\title{
Bacteriuria and bacteraemia in patients with long-term indwelling catheters-a domiciliary study
}

\author{
L. A. JEWES, W. A. GILlespie, †A. LEADBETTER, B. MYERS, R. A. SIMPSON, \\ *M. J. STOWER and A. C. VIANT
}

Departments of Microbiology, "Urology and tCommunity Nursing, Bristol and Weston Health Authority, Bristol, BS2 8HW

\begin{abstract}
Summary. Men with indwelling catheters and men and women with suprapubic catheters were studied in their homes. Urine and blood were cultured and body temperature recorded after every catheter change. Nearly all patients had infected urine after 4 weeks of catheterisation, and all had bacteriuria after longer periods, usually with a mixture of organisms. Culture on selective media revealed a wider range of organisms than was detected on routine C.L.E.D. and blood agar with antibiotic sensitivity disks, but routine culture gave adequate information for clinical purposes. Bacteraemia was demonstrated after 20 of 197 changes of urethral catheter and after one of 19 changes of suprapubic catheter; but no patient had pyrexia or other symptoms. However, two had rigors on other occasions. When assessing "risk factors" for blood-stream infection in catheterised patients, it is important to record the total incidence of bacteraemia, asymptomatic as well as symptomatic.
\end{abstract}

\section{Introduction}

Operations on the lower urinary tract that require the use of catheters or other urethral instruments are amongst the commonest causes of bacteraemia (Svanbom, 1979, Roberts, 1980). Most reports have been of studies in hospital, especially after operations in patients with infected urine. Bacteraemia may occur immediately after operation, or it may be provoked by the removal or change of catheter some days later (Slade, 1958; Talbot, 1962; Cafferkey et al., 1980, 1982). Patients with long-term indwelling catheters are usually nursed in geriatric, orthopaedic and medical wards, often have bacteriuria, and sometimes suffer from bacteraemia (Jepsen et al., 1982; Bjork et al., 1984).

Most studies of catheter-associated bacteraemia have been in patients with symptoms such as pyrexia or rigor. We have occasionally observed such bacteraemic symptoms after manipulation or change of catheter in patients with long-term bladder drainage. One such incident, which led to severe septicaemia, prompted us to undertake the present investigation.

Bacteraemia, usually transient and symptomless, can often be demonstrated during prostatectomy in patients with infected urine (Sullivan et al., 1973; Murphy et al., 1984). It seems likely that symptom-

Received 8 Sep. 1987 ; accepted 28 Sep. 1987.

Correspondence should be sent to Professor W. A. Gillespie. less, transient bacteraemia may also occur after manipulation of long-term indwelling catheters. The incidence of bacteraemia might be influenced by the nature of the bacteria in the urine, the underlying urethral abnormality and the type of catheter used. To assess the importance of these and other "risk factors", we undertook an investigation to estimate the total incidence of bacteraemia, whether symptomatic or not. This was the principal aim of our study. We also studied the bacteriology of the urine in relation to the duration of catherisation, and we assessed the value of using selective culture media in addition to those used for routine examination of urine.

\section{Patients and methods}

\section{Study population}

A total of 115 patients was studied in their homes in the Bristol area between 12 September 1984 and 17 December 1986; 102 were men with indwelling urethral catheters and 13 patients (8 men, 5 women) had suprapubic catheters. In most cases indwelling catheterisation was necessary because of lesions of the bladder, urethra or prostate, often in patients who were unfit for prostatectomy. In others, the reason was urinary retention or incontinence resulting from cerebro-vascular accidents or other neuropathic disorders (e.g., multiple sclerosis).

The urethral catheters were of the Foley type, with diameters of 14-20 Charrière gauge, retained in position 
by balloons of $5-30 \mathrm{ml}$ capacity. The wider catheters with larger balloons were used in patients with patulous urethras. Catheters were changed at intervals that varied from 2 weeks to 3 months. The same nurse (AL) changed all the catheters, and collected a blood culture immediately after each catheter change. Urine for culture was taken on each occasion. The oral body temperature was recorded with an electronic thermometer immediately before catheter change, $1 \mathrm{~h}$ afterwards, again, usually, on the same evening, and next morning. The evening temperature was taken by the patient or a relative, and read by the nurse next morning. Details of antibiotic and other treatment were recorded at the first visit, and symptoms, if any, after the catheter change were recorded on the day after the change. Any later symptoms were recorded at the next visit.

\section{Bacteriological methods}

Urine culture. All specimens were cultured and isolates identified in the routine laboratory, by a quantitative standard loop method, on C.L.E.D. medium (Cysteine Lactose Electrolyte-deficient medium; Mast Laboratories Ltd, Bootle, Merseyside) and on D.S.T. agar (Oxoid) containing $5 \%$ lysed defibrinated horse blood with antibiotic disks for direct sensitivity tests (Stokes and Ridgway, 1980). The lysed blood was supplied by Lab M, Topley House, PO Box 19, Bury, Lancs. The disks (Oxoid) contained ampicillin $25 \mu \mathrm{g}$, trimethoprim $1.25 \mu \mathrm{g}$, nitrofurantoin $50 \mu \mathrm{g}$, gentamicin $10 \mu \mathrm{g}$, nalidixic acid $30 \mu \mathrm{g}$, cephradine $30 \mu \mathrm{g}$. In addition, to investigate more precisely the incidence of mixed infections, urine specimens obtained between November 1984 and April 1986 were plated on the following selective media: Mannitol Salt Agar (BBL Microbiology Systems, Becton Dickinson UK Ltd, York House, Empire Way, Wembley, Middlesex); MacConkey Agar (Oxoid); a medium selective for Proteus and Providencia spp. (Clayton et al., 1982), containing colistin $100 \mathrm{mg} / \mathrm{L}$ and inositol $10 \mathrm{~g} / \mathrm{L}$ in Nutrient Agar Base (BBL); Pseudomonas Agar F (Difco) containing cetrimide $0.01 \%$; neomycin blood agar containing neomycin $70 \mathrm{mg} / \mathrm{L}$ in Blood Agar Base (Lab M).

The minimum viable count for diagnosis of bacteriuria was $10^{4} \mathrm{cfu} / \mathrm{ml}$. All urinary isolates were identified to genus level, with the exception of lactose-fermenting gram-negative rods which were recorded initially as "coliform bacilli", in line with the routine practice of clinical reporting in this laboratory.

During the last 6 months of the study, all gram-negative bacilli isolated from urine were identified to species level by means of the API system. This enabled our results to be compared with other published reports. Throughout the whole survey, all organisms isolated from the urine of patients with positive blood cultures were fully identified.

Blood culture. Approximately $10 \mathrm{ml}$ of blood was taken for culture, half into $45 \mathrm{ml}$ of Brain Heart Infusion Broth; (Gibco Ltd, Trident House, PO Box 35, Renfrew Road, Paisley PA3 4EF) containing sucrose $10 \%$ and polyanethol-sulphonic acid (Liquoid: Roche Products, Diagnostic Dept, PO Box 8, Welwyn Garden City, Herts
AL7 3AY) $0.5 \%$ for aerobic cultures and half into $90 \mathrm{ml}$ of anaerobic broth-Brain Heart Infusion Broth (Gibco) containing sodium mercaptoacetate $0.1 \%$, glucose $0.1 \%$, methylene blue $0.000075 \%$, and Oxoid agar no. $30.02 \%$. The blood cultures were examined daily during incubation at $37^{\circ} \mathrm{C}$ for 7 days. Subcultures were made when there was evidence of bacterial growth and routinely after 2 and 7 days; subcultures were on Blood Agar Base (Lab M) containing defibrinated horse blood (Lab M) 5\% incubated in air and $\mathrm{CO}_{2} 10 \%$ at $37^{\circ} \mathrm{C}$ and on blood agar containing yeast extract (Oxoid) $0.3 \%$ and L-cysteine hydrochloride $0.05 \%$ incubated anaerobically for $48 \mathrm{~h}$ at $37^{\circ} \mathrm{C}$. Isolates were identified by means of the API system. Antibiotic sensitivities were performed by the Stokes comparative disk method (Stokes and Ridgway, 1980). Coagulase-negative staphylococci were considered significant only when detected in both blood-culture bottles after incubation for 2 days and when similar organisms with the same antibiotic sensitivity patterns were present in the urine.

\section{Results}

Nearly all the patients had infected urine. After 8 weeks of indwelling catheterisation, all were infected except two patients who were receiving antibiotics. In the great majority, the bacteriuria was polymicrobial. This was especially evident when the urine was cultured on selective media (figure and table I). Generally, the longer the duration of catheterisation, the greater were the variety and number of bacterial species present in the urine (table II).

The use of selective media revealed the complexity of the bacteriology of the urine of catheterised patients, in agreement with the findings of Clayton et al. (1982). It did not alter the accuracy of diagnosis of infection nor of antibiotic sensitivity testing.

Among the wide variety of organisms isolated from urine (tables I and III), Escherichia coli

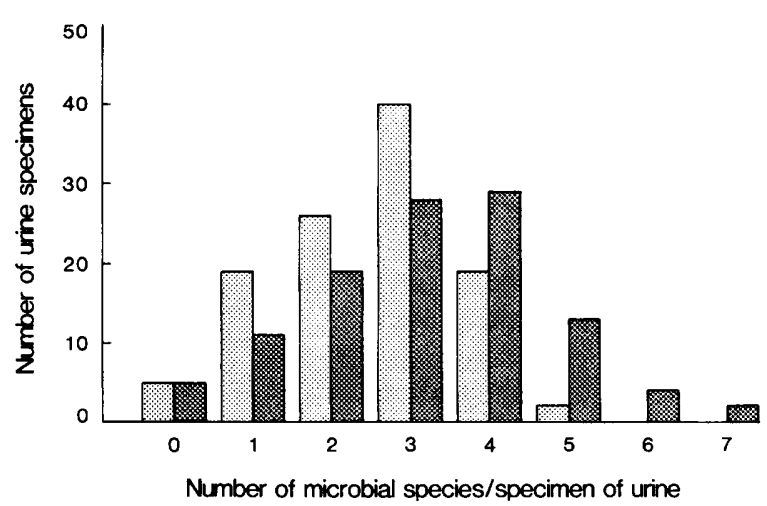

Figure. Comparison of results of urine culture on "routine" media ( 8 ) only and "routine" with selective media ( 
Table I. Organisms isolated from 122 urine specimens by use of (a) C.L.E.D. plates and blood agar with direct sensitivity disks, and (b) selective media

Number of isolates

Bacteria isolated

(a)

(b)

"Coliform bacilli" (lactose-ferment-

ing)

Proteus spp.

Pseudomonas spp.

Providencia spp.

Morganella spp.

Acinetobacter spp.

Other gram-negative rods

Streptococcus spp.

Staphylococcus aureus

Staphylococcus, coagulase-negative

Corynebacterium spp.

Candida spp.

-

was comparatively uncommon and accounted for $<15 \%$ of the gram-negative bacilli. This finding was similar to that of Kunin et al. (1987), but differed from that of a multicentre survey, in which $E$. coli was the commonest gram-negative bacillus (Jepsen et al., 1982).

Some of the urinary organisms were resistant to one or more antibiotics, often to several (table III). Most patients had been in hospital previously and many probably had received antibiotics, but only 32 patients were receiving antibiotics when the urine specimens were taken. This figure included ten patients who were taking antibiotics for reasons other than urinary-tract infection. The chemother- apy rarely cleared the bacteriuria, and then only temporarily (table II).

There were 20 episodes of bacteraemia after 197 changes of urethral catheter (an incidence of $10 \cdot 2 \%$ ). The organisms isolated from the blood cultures are shown in table IV. Four blood cultures yielded two different organisms, and one yielded three. One of the 19 changes of suprapubic catheter was followed by bacteraemia with Providencia stuartii.

No episode of bacteraemia was accompanied by any relevant symptoms, although two patients had rigors on other occasions. Body temperatures did not differ appreciably before and after catheter change, and none rose above $37 \cdot 5^{\circ} \mathrm{C}$. The temperature records of bacteraemic patients were indistinguishable from those with negative blood cultures.

\section{Predisposing factors for bacteraemia}

Bacteraemia was commoner in patients with lesions of the prostate, urethra or bladder (17 out of 137 changes) than in those with neuropathic disorders ( 3 out of 60 changes). However, the difference was not statistically significant $\left(\chi^{2}=1.764 ; p=0.092\right)$. There was no evidence that any particular urinary organism was more likely than others to enter the blood stream. There was no evidence that the incidence of bacteraemia was related to any one of the wide variety of Foley-type catheters that were used, nor to the size of the catheter-retaining balloons. It must be remembered, however, that wider catheters with larger balloons were used in patients with patulous, hence wider, urethras.

Table II. Numbers of microbial species isolated from urine cultured on routine medium, in relation to duration of catheterisation and antibiotic administration

\begin{tabular}{lccccc}
\hline & & & & \multicolumn{1}{c}{$\begin{array}{c}\text { Number of species per } \\
\text { urine specimen }\end{array}$} \\
$\begin{array}{l}\text { Duration of } \\
\text { catheterisation }\end{array}$ & $\begin{array}{c}\text { Patients receiving } \\
\text { antibiotics }\end{array}$ & $\begin{array}{c}\text { Number of urine } \\
\text { specimens }\end{array}$ & $\begin{array}{c}\text { Number of } \\
\text { positive cultures }\end{array}$ & range & median \\
\hline Less than 1 month & No & 9 & 9 & $1-3$ & 2 \\
1-6 months & Yes & 3 & 1 & $0-1$ & 2 \\
6-12 months & Yes & 45 & 43 & $0-5$ & 2 \\
1-5 years & No & 13 & 11 & $0-4$ & 3 \\
More than 5 years & No & 37 & 37 & $1-4$ & 1 \\
& Yes & 7 & 7 & $2-4$ & 3 \\
Total & No & 57 & 67 & $1-3$ & 2 \\
& Yes & 17 & 3 & $2-4$ & 3 \\
\end{tabular}


Table III. Identity and antibiotic sensitivity of gram-negative bacilli isolated from urine (JuneDecember, 1986)

\begin{tabular}{lccc}
\hline \multicolumn{1}{c}{ Species } & $\begin{array}{c}\text { Resistant to one or more } \\
\text { antibiotics to which } \\
\text { normally sensitive }\end{array}$ & $\begin{array}{c}\text { Antibiotic } \\
\text { sensitive }\end{array}$ & Total \\
\hline Escherichia coli & 8 & 12 & 20 \\
Citrobacter freundi & 3 & 8 & 11 \\
Citrobacter diversus & - & 1 & 1 \\
Klebsiella aerogenes & 5 & 10 & 15 \\
Klebsiella oxytoca & 4 & 18 & 22 \\
Klebsiella rhinoscleromatis & 1 & 0 & 1 \\
Enterobacter cloacae & 3 & 0 & 3 \\
Enterobacter amnigenus & 0 & 3 & 3 \\
Enterobacter agglomerans & 0 & 1 & 1 \\
Enterobacter aerogenes & 1 & 17 & 17 \\
Pseudomonas aeruginosa & 0 & 29 & 32 \\
Proteus mirabilis & 3 & 1 & 1 \\
Proteus vulgaris & 0 & 7 & 8 \\
Morganella morganii & 1 & 6 & 6 \\
Providencia stuartii & 0 & 2 & 2 \\
Providencia rettgeri & 0 & 3 & 4 \\
Serratia marcescens & 1 & 2 & 2 \\
Acinetobacter calcoaceticus var. anitratus & 0 & 120 & 150 \\
Total & 30 & & \\
\hline
\end{tabular}

Table IV. Bacteriuria and bacteraemia in men with indwelling urethral catheters; urine and blood samples were taken immediately after catheter changes

\begin{tabular}{lccc}
\hline $\begin{array}{c}\text { Duration of } \\
\text { catheterisation }\end{array}$ & $\begin{array}{c}\text { Total number } \\
\text { of urine } \\
\text { specimens }\end{array}$ & $\begin{array}{c}\text { Number of } \\
\text { infected urine } \\
\text { specimens }\end{array}$ & $\begin{array}{c}\text { Positive * } \\
\text { blood cultures }\end{array}$ \\
\hline <1 month & 12 & 10 & 2 \\
1-6 months & 58 & 54 & 6 \\
6-12 months & 44 & 44 & 7 \\
1-5 years & 63 & 63 & 3 \\
$>5$ years & 20 & 20 & 2 \\
All & 197 & 191 & 20 \\
\end{tabular}

* The organisms were: Proteus mirabilis 5 isolates, Proteus sp. 1, Ps. aeruginosa 4, Streptococcus faecalis 4, S. agalactiae 1, Citrobacter freundi 2, C. diversus 1, $K$. aerogenes 2, Prov. stuartii 1, Prov. rettgeri 1, Staphylococcus (coagulase-negative) 2, Ent. cloacae 1, M. morganii 1. Four blood cultures yielded two species and one yielded three.

\section{Discussion}

In the management of catheterised patients, much emphasis, rightly, is placed on the importance of aseptic precautions, including the maintenance of closed drainage. With correct catheter care, bacteriuria usually can be avoided during short periods of indwelling catheterisation; but when the catheter remains in situ for more than a few weeks, bacteriuria is almost inevitable (Slade and Gillespie, 1985; Kunin, 1987). The longer the period of catheterisation, the greater was the incidence of mixed infection by organisms many of which were resistant to several antibiotics. These facts underline the limitations of antibiotics in the management of catheter-associated urinary infection (Bjork et al., 1984; Gillespie, 1986; Kinder, 1987). It was gratifying to find that relatively few patients in our series were receiving antibiotics although many may have received them previously, in hospital or at home. By using special selective media, an even greater range of organisms could be demonstrated in the urine, confirming the findings of Clayton et al. (1982), but urine culture on selective media is unnecessary for routine diagnosis of infection and antibiotic management in catheterised patients. Our results show that adequate information is obtained by culture on C.L.E.D. agar, combined with direct antibiotic-sensitivity tests.

Bacteraemia, with symptoms such as pyrexia and rigors, sometimes fatal, is a well-recognised complication of catheterisation and urethral instrumentation (Jepsen et al., 1982; Slade and Gillespie, 1985; Kunin, 1987). The risk of bacteraemia is especially great in patients with infected urine. It is well known that catheter-associated bacteraemia may be symptomless, but the magnitude of the difference between the incidence of symptomatic and asymptomatic cases may not be widely recog- 
nised. Our findings suggest that the number of bacteraemic patients with symptoms may be a very small proportion of the total. Catheter-associated bacteraemia, even when asymptomatic, may lead to bacterial endocarditis in susceptible patients (Bayliss et al., 1984). It is clear, therefore, that an assessment of the risk factors for bacteraemia in catheterised patients requires systematic study of total incidence of bacteraemia, and should not be limited to patients with symptoms. There were several factors that we could not study for practical and ethical reasons - e.g. the use of different types and sizes of catheter chosen at random, and the skill and experience of the catheter operator. In our study, bacteraemia was commoner in patients with lesions of the prostate, urethra or bladder than in those with normal urethras who were catheterised because of neurological disorders. The incidence of

\section{REFERENCES}

Bayliss R, Clarke C, Oakley C M, Somerville W, Whitfield A G, Young S E 1984 The bowel, the genitourinary tract and infective endocarditis. British Heart Journal 51 : 339-345.

Bjork D T, Pelletier L L, Tight R R 1984 Urinary tract infections with antibiotic resistant organisms in catheterized nursing home patients. Infection Control 5: 173-176.

Cafferkey M T, Conneely B, Falkiner F R, Gillespie W A, Murphy D 1980 Post-operative urinary infection and septicaemia in urology. Journal of Hospital Infection 1 : 315320.

Cafferkey M T, Falkiner F R, Gillespie W A, Murphy D M 1982 Antibiotics for the prevention of septicaemia in urology. Journal of Antimicrobial Chemotherapy 9: 471-477.

Clayton C L, Chawla J C, Stickler D J 1982 Some observations on urinary tract infections in patients undergoing long-term bladder catheterization. Journal of Hospital Infection 3: 3947.

Gillespie W A 1986 Antibiotics in catheterized patients. Journal of Antimicrobial Chemotherapy 18: 149-151.

Jepsen O B et al. 1982 Urinary-tract infection and bacteraemia in hospitalised medical patients--a European multi-centre prevalence survey on nosocomial infection. Journal of Hospital Infection 3: 241-252.

Kinder R B 1987 Long term urethral catheterisation in the elderly. British Medical Journal 294: 792-793.

Kunin C M 1987 Detection, prevention and management of urinary tract infections, 4th edn. Lea and Febiger, Philadelphia, pp 248 and 251 .

Kunin C M, Chin Q F, Chambers S 1987 Indwelling urinary bacteraemia was not affected by the nature of the organisms in infected urine. The absence of $E$. coli from the blood cultures, at first sight surprising, was consistent with its comparative infrequency in the patients' urine. In this respect, patients with long-term catheters tend to differ from those whose catheters are removed a few days after prostatectomy (Slade and Gillespie, 1985).

Our study shows that the microbiological findings in catheter-associated urinary infection in domiciliary patients are similar to those encountered in hospital (Clayton et al., 1982; Kunin, et al., 1987).

We thank the doctors who allowed us to study their patients, and the patients and their relatives for their cooperation. We are grateful to Professor D. C. E. Speller, Mr J. B. M. Roberts, Mr P. J. B. Smith and Dr A. J. Hedges for their advice and support. We thank Mrs B. Handley for expert technical assistance.

catheters in the elderly. Relation of "catheter life" to formation of encrustations in patients with and without blocked catheters. American Journal of Medicine 82: 405411.

Murphy D M, Stassen L, Carr M E, Gillespie W A, Cafferkey M T, Falkiner F R 1984 Bacteraemia during prostatectomy and other transurethral operations: influence of timing of antibiotic administration. Journal of Clinical Pathology 37: 673-676.

Roberts F J 1980 A review of positive blood cultures: Identification and source of microorganisms and patterns of sensitivity to antibiotics. Reviews of Infectious Diseases 2: 329-339.

Slade N 1958 Bacteraemia and septicaemia after urological operations. Proceedings of the Royal Society of Medicine 51 : 331-334.

Slade N, Gillespie W A 1985 The urinary tract and the catheter: infection and other problems. John Wiley and Sons, Chichester, pp 11, 21 and 35.

Stokes E J, Ridgway, G L 1980 Clinical bacteriology, 5th edn. Edward Arnold, London, p 209.

Sullivan N M, Sutter V L, Mims M M, Marsh V H, Finegold S M 1973 Clinical aspects of bacteremia after manipulation of the genitourinary tract. Journal of Infectious Disease 127: 49-55.

Svanbom M 1979 Septicemia. I. A prospective study on etiology, underlying factors and sources of infection. Scandanavian Journal of Infectious Diseases 11 : 187-198.

Talbot C H 1962 Septicaemia due to gram-negative bacilli. Lancet 1 : 668-670. 